\title{
PGC-1 $\alpha$ activates SIRT3 to modulate cell proliferation and glycolytic metabolism in breast cancer
}

\author{
Yan ZU ${ }^{1, *}$, Xiao-Fei CHEN ${ }^{2}$, Qiang LI ${ }^{1}$, Shu-Ting ZHANG ${ }^{1}$, Li-Na SI ${ }^{1}$ \\ ${ }^{1}$ School of Mechanical and Material Engineering, North China University of Technology, Beijing, China; 2Beijing Institute of Astronautical \\ System Engineering, Beijing, China \\ *Correspondence: yanzu5689@163.com
}

Received May 30, 2020 / Accepted September 7, 2020

\begin{abstract}
Breast cancer is the leading cause of death among women. PGC-1 a plays an important role in the regulation of metabolic reprogramming in cancer cells. SIRT3 has significant implications for tumor growth. In this study, we explored the roles of PGC-1 $\alpha$ and SIRT3 in cell proliferation and mitochondrial energy metabolism alterations in breast cancer cells. The expression patterns of PGC-1 $\alpha$ and SIRT3 were examined using qRT-PCR and western blotting analysis. MCF-7 and MDA-MB231 cells were infected with adenovirus to overexpress or knock down the expression of PGC-1a and SIRT3. Cell viability and apoptosis were analyzed by CCK-8 and flow cytometry, respectively. Hexokinase 2, pyruvate kinase activities, as well as $\mathrm{NAD}^{+} / \mathrm{NADH}$ ratio and ATP concentration, were assessed by commercial kits. Glucose consumption was measured using the glucose oxidase method and lactic acid concentration was detected by lactate dehydrogenase kit. Expression levels of PGC-1 and SIRT3 were much lower in breast cancer patients, compared with the normal controls. Overexpression of PGC-1 $\alpha$ or SIRT3 both significantly promoted the apoptosis and inhibited the proliferation in MCF-7 and MDA-MB-231 cells. Additionally, PGC-1 $a$ or SIRT3 also induced the inhibition of glycolysis metabolism. Moreover, the expression of SIRT3 was positively regulated by PGC-1 $\alpha$. Silencing SIRT3 partly reversed the negative effects of PGC-1 $\alpha$ on glycolytic metabolism. These findings demonstrated that PGC-1 $\alpha /$ SIRT3 regulated cell proliferation and apoptosis of breast cancer through altering glycolysis, which may provide novel therapeutic strategies for breast cancer.
\end{abstract}

Key words: PGC-1 $\alpha$, SIRT3, breast cancer, glycolysis, metabolism

Breast cancer is currently the most prevalent neoplastic disease that threatens women's health [1]. Statistical analysis shows that the incidence rate of breast cancer was the highest among new cancers in women, which account for more than $30 \%$ [2]. Despite much progress has been made in the development of therapeutic strategies, the survival rate of breast cancer has not improved in recent decades [3]. Therefore, it is an urgent need to identify novel biomarkers for the diagnosis, treatment, and better understanding of the potential mechanism of breast cancer tumorigenesis.

In normal cells, energy is mainly generated from the mitochondrial oxidative phosphorylation (OxPhos) pathway [4]. However, in the tumor environment, the oxidative phosphorylation and mitochondrial respiratory function of tumor cells are irreversibly damaged. Whereas, glycolysis, the main energy acquisition method instead of OxPhos in most cancer cells even under aerobic concentrations, can be activated when the mitochondrial respiratory function is impaired, which is known as the Warburg effect
[5]. Such metabolic alterations from OxPhos to glycolysis could meet the increasing demand for energy of rapidly proliferating cancer cells, as well as protect the cancer cells from oxidative stress since mitochondria are the main source of intracellular ROS, which is a fundamental property of cancer cells [6].

Peroxisome proliferator-activated receptor-gamma coactivator 1 a (PGC-1 $\alpha$ ) is the most extensively studied member from transcriptional coactivators, which could regulate the cell survival and proliferation of cancer cells by controlling mitochondrial biogenesis, and cellular metabolism [7]. Accumulating evidence has indicated the involvement of PGC-1 $\alpha$ in the control of metabolic reprogramming in many kinds of cancer cells [8-10]. Recent studies have shown PGC-1 $\alpha$ is both a tumor suppressor and promoter in cancer development [11]. In several cancers such as prostate cancer, PGC-1 a could suppress cancer metastasis [12, 13]. However, deeper insights are still missing for the role of PGC1- $\alpha$ in breast cancer. 
Sirtuins, a family of $\mathrm{NAD}^{+}$-dependent histone deacetylases (HDACs), play essential roles in numerous physical processes including cell survival, apoptosis, metabolism, stress responses, cancer, and aging $[14,15]$. Among them, Sirtuin3 (SIRT3) is the most well-characterized mitochondrial deacetylase, which targets many enzymes that are involved in cellular metabolism, which is similar to PGC-1a. Besides, SIRT3 serves as an important regulator of energy homeostasis, and SIRT3 could coordinate the deacetylation of multiple mitochondrial proteins to regulate the mitochondrial activity, which has significant implications for tumor growth [16-18]. Previous records have shown that PGC-1a could interact directly with SIRT1 [19], and PGC-1a overexpression could upregulate the expression of SIRT3 in brown adipocytes [20]. However, the relationship between PGC-1 $\alpha$ and SIRT3 and their roles in the proliferation of breast cancer is still unclear.

In the present study, we explored the potential roles of PGC-1 $\alpha$ and SIRT3 in the glycolytic metabolism of breast cancer cells. Our results revealed that overexpression of PGC-1a/SIRT3 decreased cell proliferation and induced apoptosis by inhibiting the glycolysis in breast cancer cells.

\section{Materials and methods}

Tissues. The 30 paired breast cancer and the adjacent normal tissue samples used in this study were obtained from the tissue bank at the North China University of Technology. The samples were frozen immediately after being surgically resected from the patients and stored in liquid nitrogen. All tissues were confirmed histologically and all patients received no chemotherapy or radiotherapy before the study. The study was approved by the Ethics Committee of the North China University of Technology.

Cancer cell culture and transfection. MCF-7 and MDA-MB-231, human breast cancer cell lines, as well as normal cell line MCF10A, were purchased from the Cell Bank of the Chinese Academy of Sciences (Shanghai, China). Cells were cultured in Dulbecco's minimal Eagle's medium (DMEM; Invitrogen, Carlsbad, CA, USA). The media were supplemented with $10 \%$ fetal bovine serum (FBS; Gibco, Grand Island, NY, USA) and 10,000 U penicillin/streptomycin. The cells were incubated at $37^{\circ} \mathrm{C}$ in a humidified atmosphere containing $5 \% \mathrm{CO}_{2}$.

For cell transfection, adenoviral vectors expressing SIRT3targeting shRNA (Ad-sh-SIRT3), Ad-sh-PGC-1 $\alpha$, and SIRT3 overexpression adenoviral vector (Ad-SIRT3), Ad-PGC-1 $\alpha$, as well as negative controls (NC) were all constructed and purchased from Fenghui Biotechnology (Changsha, China). After reaching 60-70\% confluence, MCF-7 and MDA-MB231 cells were transfected with the above vectors using Polyjet $^{\mathrm{m} \text { t }}$ transfection reagent (SignaGen Laboratories, Gaithersburg, MD, USA), according to the manufacturer's protocols. To inhibit glycolysis, glycolysis inhibitor 2-DG (10 nM) was used to treat the cells before transfection.
Cell viability assay. Cell viability was assessed using the CCK-8 assay. Briefly, cells were seeded at a density of $2.5 \times$ $10^{5}$ in 96-well culture plates and were cultured overnight. After treatment of CPF for $24 \mathrm{~h}$, cells were further cultured and cell viability was measured at $6 \mathrm{~h}, 12 \mathrm{~h}, 24 \mathrm{~h}$, and $48 \mathrm{~h}$. For the CCK- 8 assay, $10 \mu \mathrm{l}$ of CCK- 8 solution was added to each well and cells were incubated at $37^{\circ} \mathrm{C}$ for another $1 \mathrm{~h}$. The absorbance of the solutions was detected at $450 \mathrm{~nm}$ by a SYNERGY-HT multiwell plate reader (Synergy HT, Winooski, VT, USA).

Flow cytometry of cell apoptosis. Apoptosis was determined by staining with Annexin V/PI (Beyotime, Haimen, China) as previously described. In brief, MCF-7 and MDA-MB-231 cells were incubated at a density of $2 \times 10^{5} /$ well in a 6-well plate with corresponding treatments, the cells were then washed with ice-cold PBS twice after removing the culture medium, and centrifuged at $800 \mathrm{rpm}$ for $5 \mathrm{~min}$, then stained with $5 \mu$ l of Annexin V and $5 \mu$ of PI incubated with $100 \mu$ of binding buffer for $15 \mathrm{~min}$ at RT in the dark. The fluorescent signal was further analyzed by flow cytometry after the reaction volume was raised to $500 \mu \mathrm{l}$ by adding binding buffer.

Hexokinase 2 and pyruvate kinase activity assay. Hexokinase 2 and pyruvate kinase activities were determined using the Hexokinase 2 Activity Assay Kit and pyruvate kinase Activity Assay Kit (Abcam, Cambridge, UK) separately as previously described and followed by a fluorometric measurement (Ex/Em 540/590 nm).

Glucose consumption assay. Glucose consumption was measured using the glucose oxidase method as reported elsewhere [21]. Intracellular glucose was calculated by subtracting the glucose concentration in the medium from the total glucose concentration in blank wells.

Lactic acid concentration. For measurement of lactic acid concentration, cell supernatant was collected after centrifugation at $6000 \times \mathrm{g}$ for $10 \mathrm{~min}$ and the lactic acid concentration was determined by a lactate dehydrogenase (LD) kit (Nanjing Jiancheng biology Co., Ltd, Nanjing, China).

$\mathrm{NAD}^{+} / \mathrm{NADH}$ analysis. The ratio of $\mathrm{NAD}^{+} / \mathrm{NADH}$ was determined by a NAD/NADH fluorescence detection kit (Colorimetric, Abcam, USA) according to the manufacturer's instruction.

ATP concentration. The mitochondrial ATP concentration was measured using the ATP determination kit according to the manufacturer's instruction (Invitrogen, USA). The ATP concentration was normalized to total protein [22].

RNA extraction and quantitative real-time PCR (qRT-PCR). Briefly, total RNA was extracted using a TRIzol ${ }^{\mathrm{mw}}$ Plus RNA Purification Kit (Thermo Fisher Scientific, USA), and cDNA was generated by $500 \mathrm{ng}$ RNA/sample using PrimeScript $^{\text {mi }}$ II 1st Strand cDNA Synthesis Kit (Takara, Dalian, China) according to the manufacturer's protocols. Quantitative real-time PCR (qRT-PCR) was performed using an ABI 7000 Prism Step One plus detection system (Life Technologies, USA). The primer sequences used for qRT-PCR as follows: 
hsa-PGC-1a-F 5'-CACCAGCCAACACTCAGCTA-3'; hsa-PGC-1 $\alpha$-R 5'-GTGTGAGGAGGGTCATCGTT-3'; hsa-SIRT3-F 5'-CGTTGTGAAGCCCGACATTG-3'; hsa-SIRT3-F 5'-CGTTGTGAAGCCCGACATTG-3'; hsa- $\beta$ actin-F 5'-AGAAGGCTGGGGCTCATTTG-3'; hsa- $\beta$ actin-R 5'-AGGGGCCATCCACAGTCTTC-3'.

The gene expression levels were normalized using $\beta$-actin as an internal reference gene, and the average relative changes were calculated using the $2^{-\Delta \Delta \mathrm{Ct}}$ method.

Western blotting analysis. After the treatments, cells were harvested and extracted into the cell lysis buffer supplemented with protease inhibitor (Beyotime, China) on ice. The cell lysates were centrifuged at 12,000 rpm for $20 \mathrm{~min}$ at $4{ }^{\circ} \mathrm{C}$ after being sonicated. Then the supernatants were collected and protein concentration was determined using the BCA protein assay kit (Beyotime, China). Protein samples were diluted in $5 \times$ SDS-PAGE loading buffer and heated at $95^{\circ} \mathrm{C}$ for $5 \mathrm{~min}$, then $25 \mu \mathrm{g}$ of protein was separated with $12 \%$ SDS-PAGE gels and transferred onto polyvinylidene fluoride (PVDF) membranes. The membranes were blocked with $5 \%$ nonfat milk powder dissolved in Trisbuffered saline containing and $0.1 \%$ Tween 20 (TBST) for $1 \mathrm{~h}$ at RT then incubated with primary antibodies at $4^{\circ} \mathrm{C}$ overnight. Primary antibodies were anti-PGC-1a (1:1000 dilution; \#2178), and anti-SIRT3 (1:1000 dilution; \#2627) (both purchased from Cell Signaling Technology). The membranes were then washed three times with TBST and incubated with secondary antibody for $1 \mathrm{~h}$ at RT. Images were captured and visualized according to the chemiluminescence system using the EU-88 image scanner. Signals were quantified and analyzed using the Image-Pro Plus 6.0 software (Media Cybernetics, Sarasota, USA).

Statistical analysis. Statistical analysis was performed using SPSS 22.0 software (IBM Corporation, NY, USA). The results were expressed as mean \pm standard deviation (SD). Comparisons were performed using one-way analysis of variance (ANOVA) and least-significant difference test. Differences were regarded as significant at $\mathrm{p}<0.05$.

\section{Results}

Overexpression of PGC-1a or SIRT3 inhibited the proliferation of breast cancer cells. First, expression patterns of PGC-1 $\alpha$ and SIRT3 were determined in the breast cancer tissues and cell lines. The results revealed that both mRNA and protein levels of PGC-1 $\alpha$ and SIRT3 were downregulated in breast cancer tissues (Figure 1A). Besides, the expression of PGC-1 $\alpha$ and SIRT3 was positively correlated in breast cancer tissues (Figure 1B). As shown in Figure $1 \mathrm{C}$, the expression of PGC-1 $\alpha$ and SIRT3 in breast cancer cell lines MCF-7 and MDA-MB-231 cells was remarkably lower in normal cell line MCF10A cells. Then, to explore the roles of PGC-1 $\alpha$ and SIRT3 in breast cancer, MCF-7 and MDA-MB-231 cells were both infected with Ad-PGC-1a or Ad-SIRT3, respectively. The data presented the high efficiency of transfection (Figures 2A, 2B). Cell viability was performed by the CCK- 8 assay and the data showed that PGC-1a or SIRT3 overexpression significantly inhibited proliferation in MCF-7 and MDA-MB-231 cells (Figure 2C). Moreover, PGC-1 $a$ or SIRT3 overexpression had no significant effect on the cell viability of MCF10A (Supplementary Figure S1A), which provides evidence that PGC-1a/SIRT3mediated proliferation repression was only to cancer cells. Besides, PGC-1a or SIRT3 knockdown by their specific shRNA both notably promoted the cell viability of MCF-7 and MDA-MB-231 cells (Supplementary Figure S1B). In addition, cell apoptosis by flow cytometry also verified that the overexpression of PGC-1a or SIRT3 markedly increased the apoptotic rate compared with the control group (Figure 2D). These findings implied that PGC-1 $\alpha$ or SIRT3 might exert the tumor suppressive function in the development of breast cancer.

Overexpression of PGC-1 $\alpha$ or SIRT3 inhibited glycolytic metabolism in breast cancer cells. Due to the key roles of PGC-1 $\alpha$ and SIRT3 in the regulation of energy metabolism, we then wondered how was the glycolysis metabolism pattern influenced by PGC-1 $\alpha$ and SIRT3 in breast cancer cells. We determined the activities of hexokinase 2 and pyruvate kinase, which are two important enzymes involved in glycolysis. The results suggested that both overexpression of PGC-1 $\alpha$ and SIRT3 could significantly decrease the activities of hexokinase 2 and pyruvate kinase in MCF-7 and MDA-MB-231 cells compared with the control group (Figure 3A). Meanwhile, glucose consumption and lactic acid concentration were also examined. We also found that overexpression of PGC-1a or SIRT3 remarkably reduced glucose consumption and lactic acid production (Figures 3B, 3C). Besides, overexpression of PGC-1 $\alpha$ or SIRT3 increased the ratio of $\mathrm{NAD}^{+}$/ $\mathrm{NADH}$ and decreased the ATP concentration in breast cancer cells (Figures 3D, 3E). All these results illuminated both PGC-1a or SIRT3 might decrease the proliferation of breast cancer cells via inhibiting glycolytic metabolism in breast cancer cells.

PGC-1 a positively regulated the expression of SIRT3. Then, we further investigated the underlying mechanism involving the inhibitory effects of PGC-1 $\alpha$ on breast cancer cells. The mRNA and protein levels of PGC-1 $\alpha$ and SIRT3 were determined in cells transfected with Ad-PGC-1 $\alpha$ or sh-PGC-1a. The results showed that the upregulation of PGC-1a significantly increased both mRNA and protein levels of SIRT3, compared with the control group (Figures 4A, 4B). However, the silencing PGC-1a led to opposite results, with a remarkably decreased level of SIRT3 (Figures 4C, 4D), suggesting that PGC-1 a could positively regulate the expression of SIRT3. However, SIRT3 overexpression did not exert any effect on PGC-1a expression (Supplementary Figure S1C). These data suggested that SIRT3 might act as a downstream effector of PGC-1a. 

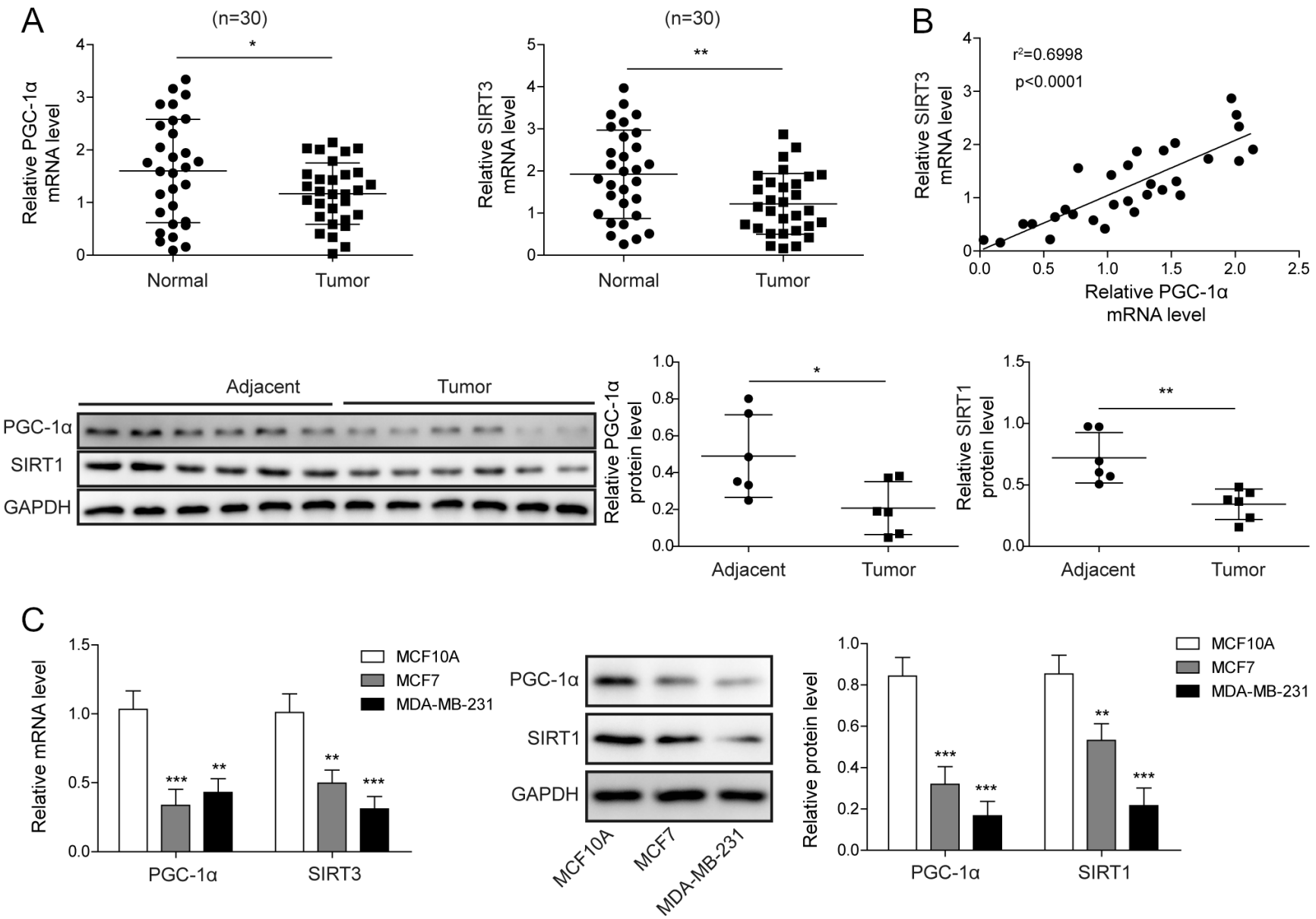

Figure 1. Effects of PGC-1 $a$ or SIRT3 overexpression on breast cancer cells. A) mRNA and protein expression levels of PGC-1 $\alpha$ or SIRT3 were determined in breast cancer tissues by RT-qPCR and western blotting, respectively. B) The correlation between PGC-1 $\alpha$ and SIRT3 in breast cancer tissues. C) Expression of PGC-1 $\alpha$ and SIRT3 in normal cell line MCF10A and breast cancer cell lines was determined by western blotting and qRT-PCR. Data are presented as mean \pm SD. Statistical significance compared with the control is indicated by ${ }^{\star} p<0.05,{ }^{\star *} p<0.01$, and ${ }^{\star * *} p<0.001$.

SIRT3 is involved in PGC-1 $a$-induced proliferation of breast cancer cells. To further illuminate the underlying mechanisms for the interaction between SIRT3 and PGC-1a in breast cancer, we infected MCF-7 and MDA-MD-231 cells with adenoviral vectors to upregulate PGC- $1 \alpha$ and meanwhile silencing SIRT3 expression. As shown in Figures 5A and 5B, the expression of SIRT3 was remarkably enhanced by the overexpression of PGC-1 $\alpha$. However, the silencing SIRT3 has little effect on the PGC-1 $\alpha$ level, indicating that SIRT3 was a downstream protein of PGC-1a. Further, the knockdown of SIRT3 partially reversed the inhibition of hexokinase 2 and pyruvate kinase activity by PGC-1 $1 \alpha$ overexpression (Figure 5C). Meanwhile, the glucose consumption and lactic acid production were also remarkably elevated by SIRT3 downregulation, compared with the Ad-PGC-1a group (Figures 5D, 5E). Additionally, silencing SIRT3 also significantly decreased $\mathrm{NAD}^{+} / \mathrm{NADH}$ ratio while increased ATP concentration compared with the Ad-PGC-1 $\alpha$ group (Figures 5F, 5G).
Next, we detected the effect of inhibition of SIRT3 in PGC-1 $\alpha$ overexpression cells on both cell viability and apoptosis. As expected, cell viability was significantly increased in co-transfection with Ad-sh-SIRT3 and Ad-PGC-1a cells compared to cells transfected with Ad-PGC-1 $\alpha$ alone, and this effect was reversed by the glycolysis inhibitor 2-DG (Figure 5H). Moreover, PGC-1a knockdown could also rescue the repression of proliferation induced by SIRT3 overexpression in MCF-7 and MDA-MD231 cells (Supplementary Figure S1D). On the contrary, inhibition of SIRT3 remarkably decreased the cell apoptosis rate in co-transfection with Ad-sh-SIRT3 and Ad-PGC-1a cells compared with cells transfected with Ad-PGC-1a alone, and this effect was also reversed by treatment of 2-DG (Figure 4I). Taken together, these results illuminated that PGC-1 $\alpha$ suppressed glycolytic metabolism by activating SIRT3, thereby inhibiting breast cancer cells' proliferation and inducing apoptosis. 


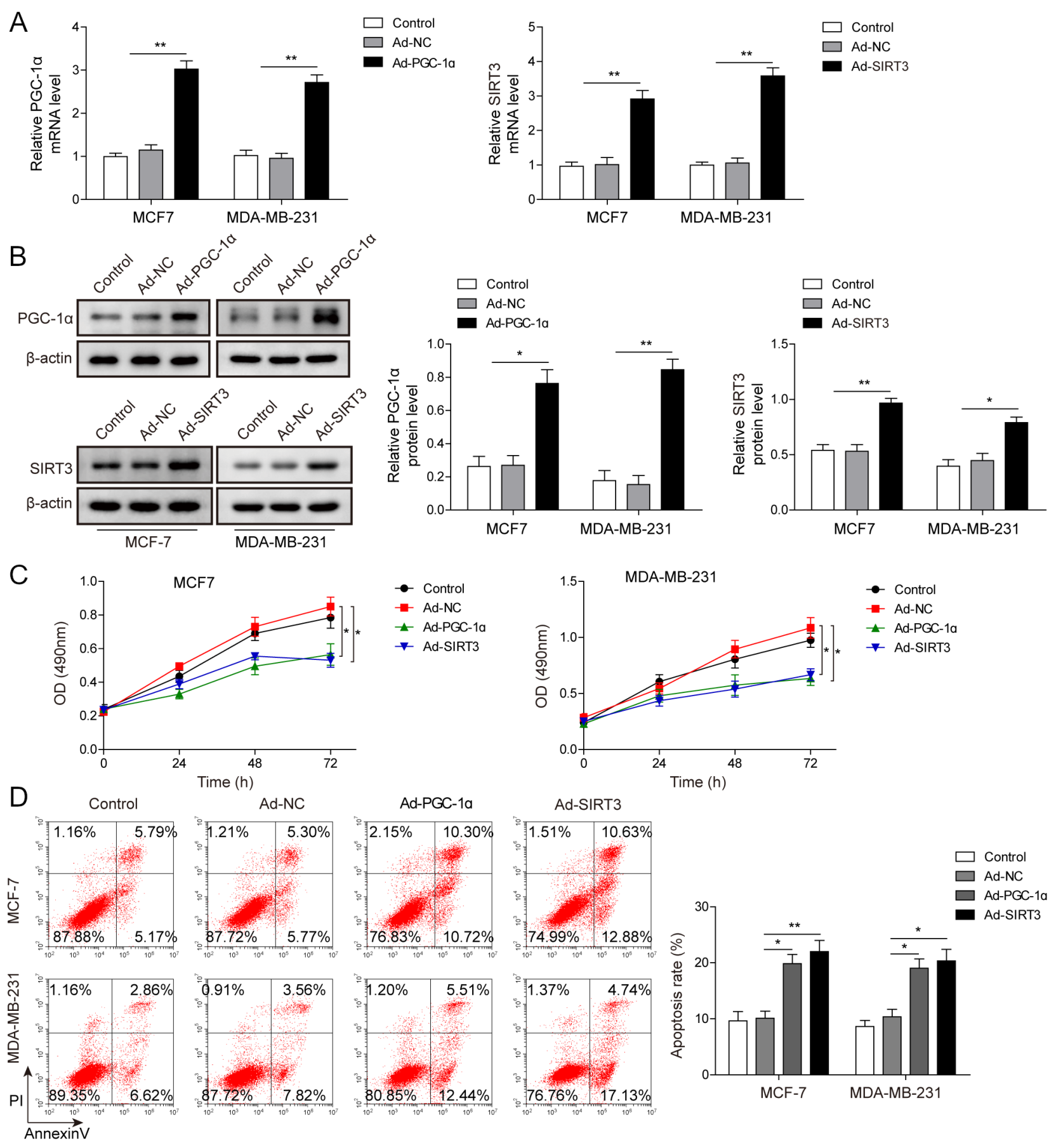

Figure 2. Effects of PGC-1 $\alpha$ or SIRT3 overexpression on breast cancer cells. A) mRNA expression of PGC-1 $\alpha$ or SIRT3 by qRT-PCR in cells transfected with Ad-SIRT3, or Ad-NC. B) Protein expression of PGC-1 $\alpha$ or SIRT3 by western blotting in cells transfected with Ad-SIRT3, or Ad-NC. C) Cell viability was determined by the CCK-8 assay. D) Cell apoptosis rate was determined with Annexin V-FITC/PI staining by flow cytometry. Data are presented as mean $\pm \mathrm{SD}$. Statistical significance compared with the control is indicated by ${ }^{\star} \mathrm{p}<0.05,{ }^{\star *} \mathrm{p}<0.01$, and ${ }^{\star * *} \mathrm{p}<0.001$.

\section{Discussion}

Breast cancer is now the leading cause of death among female patients worldwide [23]. In order to support the rapid growth and proliferation, numerous cancer cells including breast cancer cells utilize aerobic glycolysis rather than oxidative phosphorylation as the main energy source, namely the Warburg effect $[24,25]$. Altered energy metabolism plays an essential role in the development of cancer. In this paper, we determined the relationship between PGC-1 $\alpha$ 
and SIRT3, and how they contributed to the development of breast cancer cells. Our results revealed that PGC-1a could suppress glycolytic metabolism by activating SIRT3 to moderate the proliferation of breast cancer cells.

PGC-1 $\alpha$, a member of the transcriptional co-activators family [26], plays a key role in the regulation of cellular metabolism and cancer development [27-30]. The relationship between PGC-1 $\alpha$ and breast cancer aggressiveness remains controversial. Some studies have demonstrated that the expression of PGC-1 $\alpha$ is enriched in breast cancer cells $[31,32]$. However, PGC-1 $\alpha$ also promoted the sensitivity of anti-folate therapy in breast cancer [33]. In most cancers, PGC-1 $\alpha$ is considered as a tumor suppressor rather than a promoter. It has been reported that PGC-1a could suppress the cell metastasis in prostate cancer, and the expression of PGC-1 1 led to the impaired tumor growth and enhanced sensitivity to cytotoxic therapies in clear cell renal cell carcinoma $[12,34]$. In this study, we found that the overexpression of PGC-1a led to a significant decrease in cell viability and an increase of the apoptotic rate of breast cancer cells via inhibiting glycolysis. Furthermore, the inhibition of SIRT3 led to the opposite result, which was reversed by glycolysis inhibitor 2-DG.

It is widely accepted that most cancer cells change the traditional cellular metabolism to use mainly glycolysis rather than oxidative phosphorylation to gain cellular energy [35]. In glycolysis, hexokinase 2 catalyzes the phosphorylation of glucose to glucose-6-phosphate at the initial step. Selective inhibition of hexokinase 2 activity could reduce the produc- tion of glucose-6-phosphate, thereby blocking the glycolysis and inhibiting the proliferation of cancer cells [36, 37]. Pyruvate kinase catalyzes the transfer of a phosphate group from phosphoenolpyruvate (PEP) to adenosine diphosphate (ADP) at the final step of glycolysis [38]. Lack of pyruvate kinase could slow down the process of glycolysis [39]. And overexpression of pyruvate kinase was observed in many tumors, leading to the shift of energy metabolism towards glycolysis [40]. In the present study, we observed that the overexpression of PGC-1 $\alpha$ significantly decreased hexokinase 2 and pyruvate kinase activities, as well as decreased the levels of glucose consumption, ATP concentration, and lactic acid production, and increased the level of $\mathrm{NAD}^{+} / \mathrm{NADH}$ ratio in breast cancer cells. In contrast, hexokinase 2 and pyruvate kinase activities were increased by the inhibition of SIRT3 in breast cancer cells, demonstrating that PGC-1a exerts a potential role in enhancing glycolysis in breast cancer cells through the regulation of SIRT3.

SIRT3, a major mitochondrial deacetylase, plays an important role in cellular metabolism [15]. Similar to PGC-1 1 , SIRT3 has been suggested to be both a tumor promoter and suppressor [41]. It was found that an elevated level of SIRT3 was associated with many kinds of cancers, including oral cell carcinoma and node-positive breast cancer [42, 43]. However, studies also found that SIRT3 could suppress cancer development in chronic myelogenous and hepatoma carcinoma [44]. Our data suggested that the knockdown of PGC-1 $\alpha$ could inhibit the expression of SIRT3, and PGC-1 $\alpha$ overexpression could stimulate
A

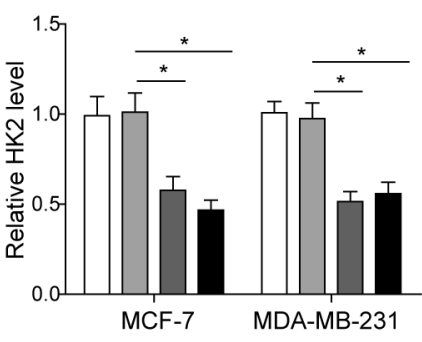

C

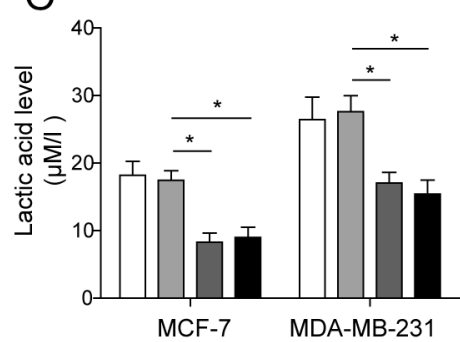

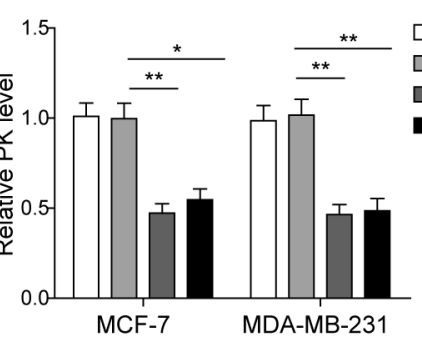

D

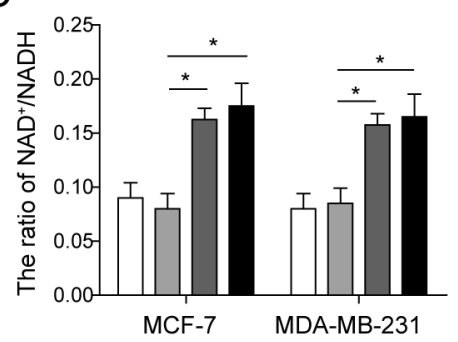

$\mathrm{B}$

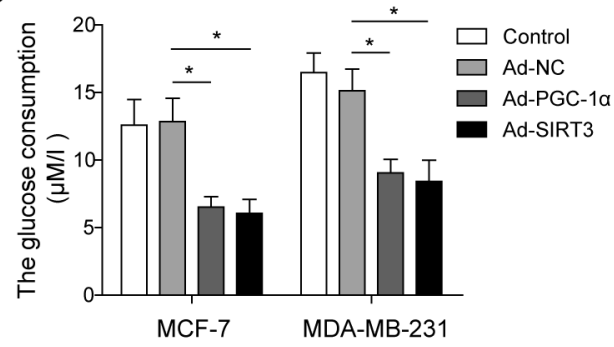

E

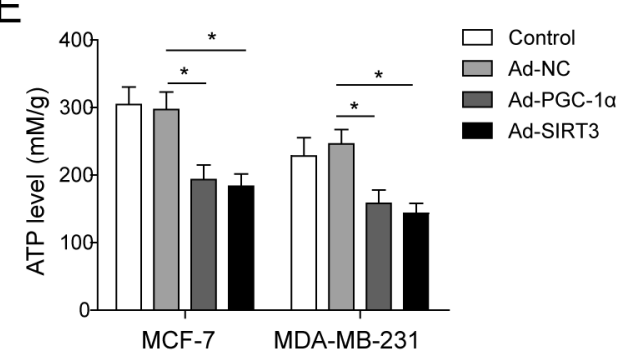

Figure 3. Effects of PGC-1 $\alpha$ or SIRT3 on glycolytic metabolism in breast cancer cells. A) Hexokinase 2 and pyruvate kinase activities were determined as described in the Materials and Methods. B) Glucose consumption was measured using the glucose oxidase method. C) Lactic acid concentration was determined by an LD kit. D) NAD ${ }^{+} / \mathrm{NADH}$ ratio and $\left.\mathrm{E}\right) \mathrm{ATP}$ concentration were measured using commercial kits. $\beta$-actin Data are presented as mean \pm SD. Statistical significance compared with the control is indicated by ${ }^{*} \mathrm{p}<0.05$ and ${ }^{* *} \mathrm{p}<0.01$. 

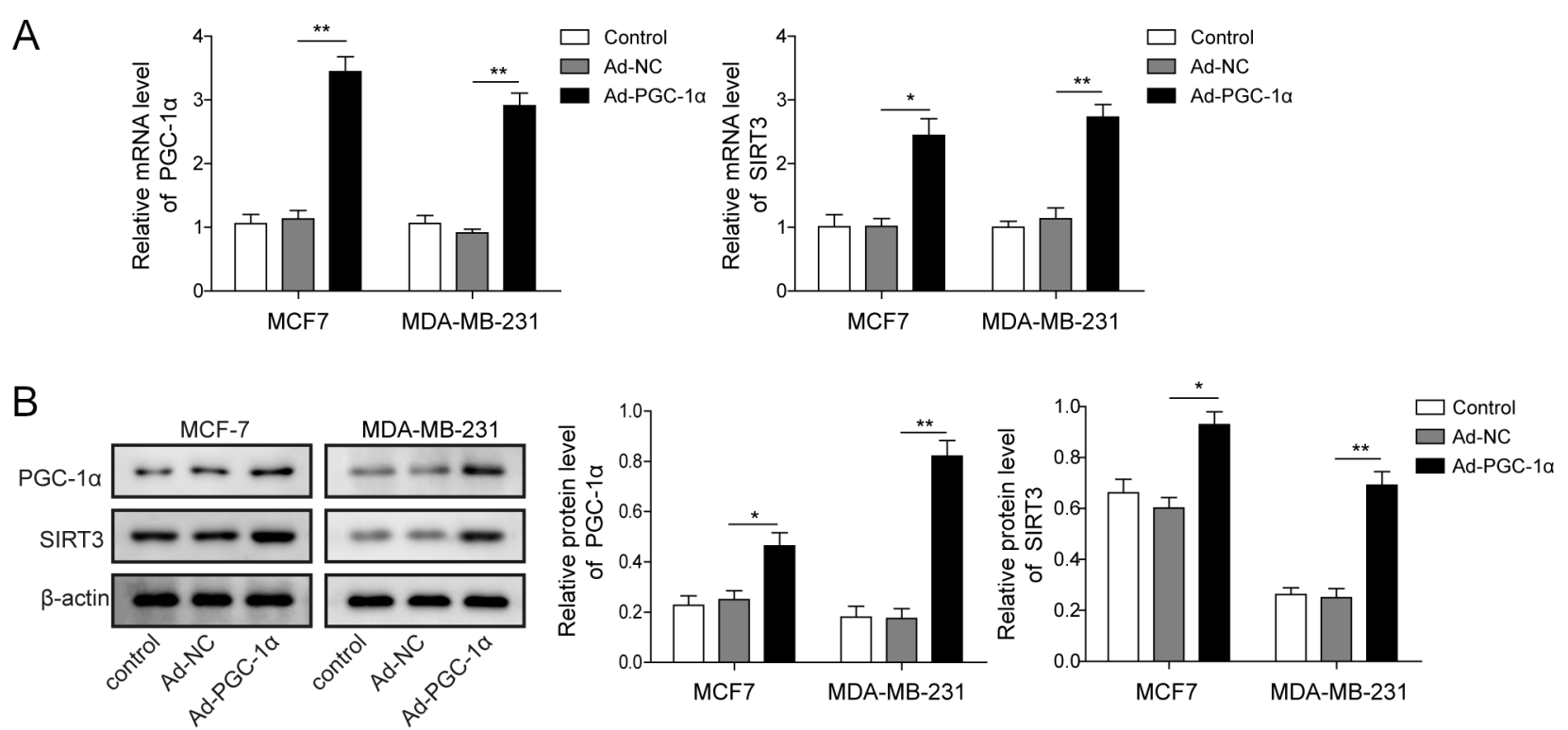

C
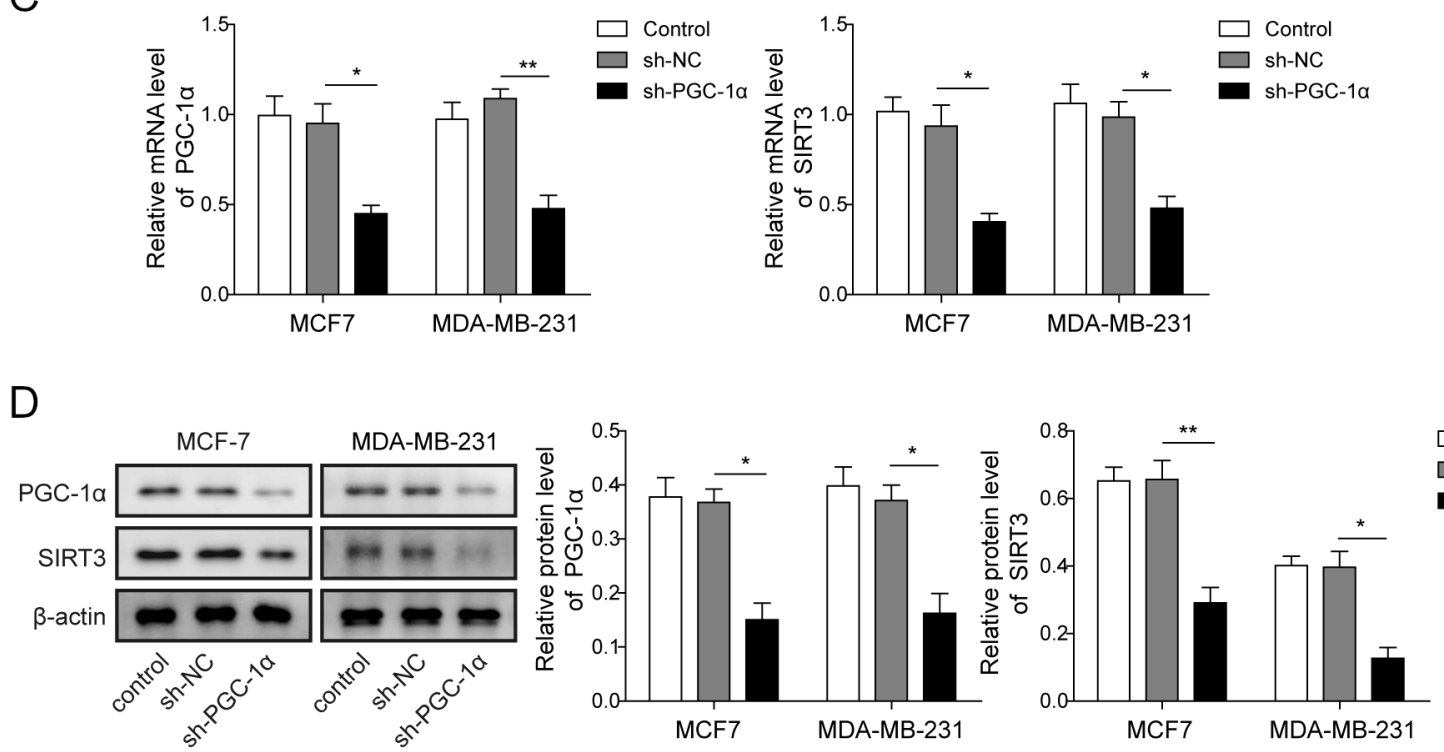

Figure 4. Overexpression of PGC-1 $\alpha$ increased the expression of SIRT3. A) mRNA and B) protein levels of PGC-1 $\alpha$, SIRT3, and $\beta$-actin (loading control) were determined in cells transfected with Ad-PGC-1 $\alpha$ by qRT-PCR and western blotting, respectively. C) mRNA expressions and D) protein levels of PGC-1 $\alpha$, SIRT3, and $\beta$-actin (loading control) were determined in cells transfected with Ad-sh-PGC-1 $\alpha$ by qRT-PCR and western blotting, respectively. Data are presented as mean \pm SD. Statistical significance compared with the control is indicated by ${ }^{*} p<0.05$ and ${ }^{\star *} p<0.01$.

the expression of SIRT3, suggesting the positive regulation of PGC-1a on SIRT3. Several studies also showed the relationship between PGC-1 $\alpha$ and SIRT3. It has been demonstrated that PGC-1 $\alpha$ could regulate the expression of SIRT3 in diabetes, ischemia-induced oxidative stress, and mitochondrial biogenesis [45-47]. In our findings, we further presented that the knockdown of SIRT3 could partly reverse PGC-1 $\alpha$-mediated glycolysis in breast cancer cells.

In conclusion, our results revealed that PGC-1a could activate SIRT3 to suppress cell proliferation by inhibited glycolytic metabolism, which may serve as a potential therapeutic target in breast cancer in the future. Meanwhile, the current study also has some limitations. In tissue samples, we didn't distinguish the subtypes of breast cancer. In in vitro studies, more underlying molecular mechanisms for the PGC-1 $1 \alpha /$ SIRT3 axis in breast cancer are still unclear and need further research to reveal them.

Supplementary information is available in the online version of the paper. 

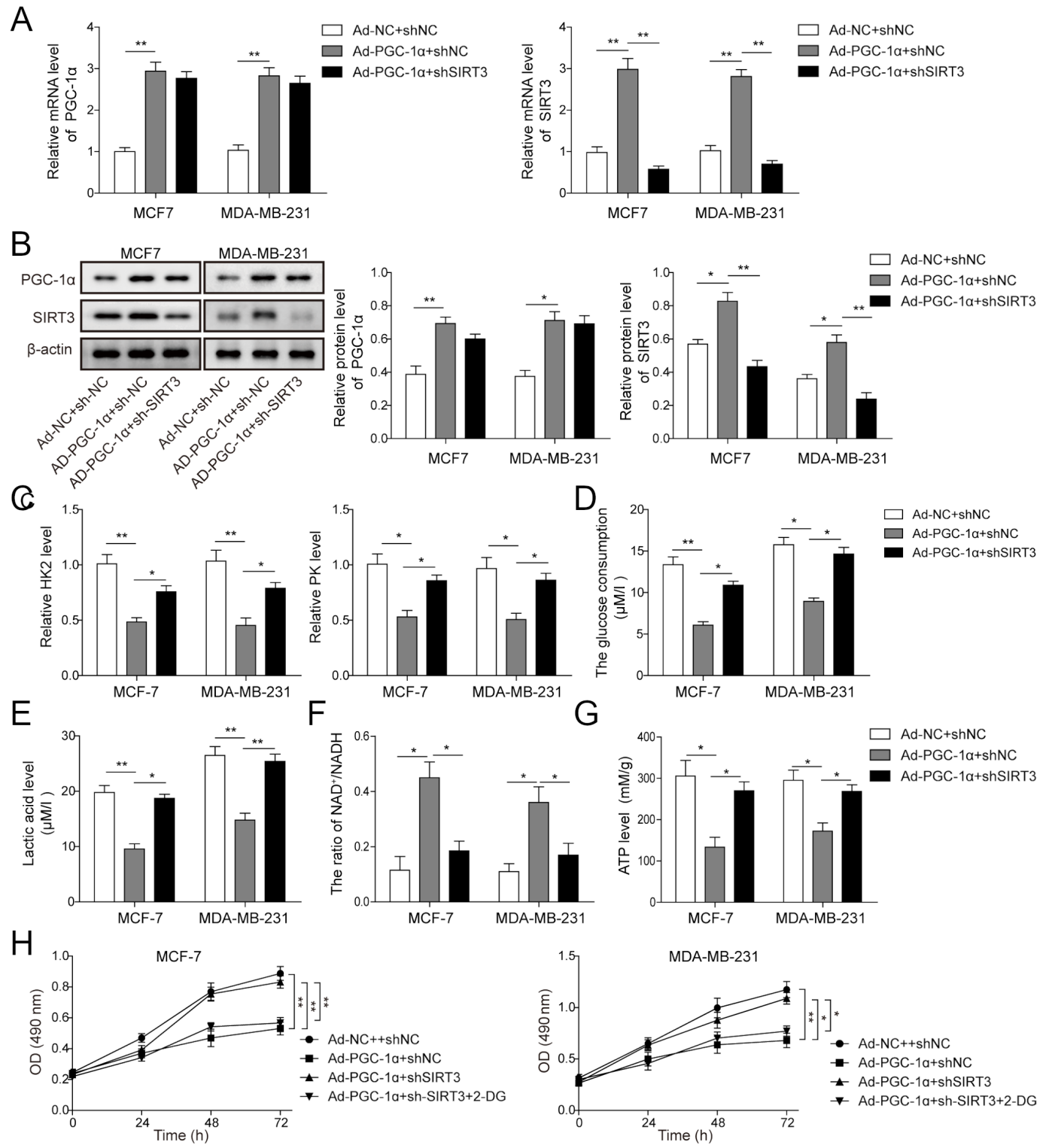

I
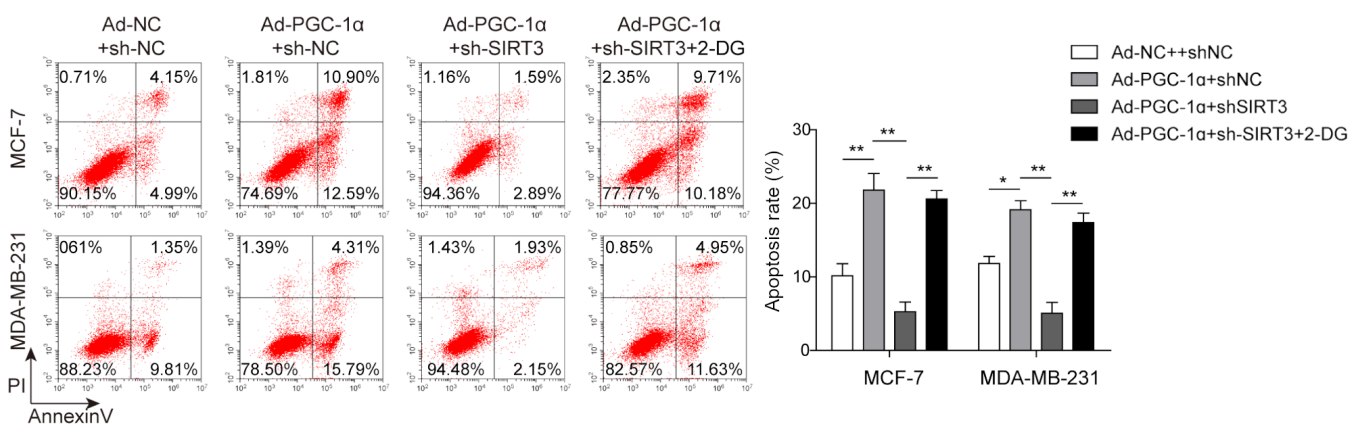

Figure 5. SIRT3 is involved in PGC-1 $\alpha$-induced proliferation of breast cancer cells. A) mRNA and B) protein expression levels of PGC-1a, SIRT3, and $\beta$-actin (loading control) were determined by RT-qPCR or western blotting. C) Hexokinase 2 and pyruvate kinase activities were determined as described in the Materials and Methods. D) Glucose consumption was measured using the glucose oxidase method. E) Lactic acid concentration was determined by an LD kit. F) $\mathrm{NAD}^{+} / \mathrm{NADH}$ ratio and G) ATP concentration were measured using commercial kits. H) Cell viability was determined by the CCK-8 assay. I) Cell apoptosis rate was determined with Annexin V-FITC/PI staining by flow cytometry. Data are presented as mean \pm SD. Statistical significance compared with control, ${ }^{*} \mathrm{p}<0.05,{ }^{* *} \mathrm{p}<0.01$. 


\section{References}

[1] GIUNTI G, GIUNTA DH, GUISADO-FERNANDEZ E, BENDER JL, FERNANDEZ-LUQUE L. A biopsy of Breast Cancer mobile applications: state of the practice review. Int J Med Inform 2018; 110: 1-9. https://doi.org/10.1016/j.ijmedinf.2017.10.022

[2] JEMAL A, SIEGEL R, WARD E, HAO Y, XU J et al. Cancer statistics, 2009. CA Cancer J Clin 2009; 59: 225-249. https:// doi.org/10.3322/caac.20006

[3] HONG CC, AMBROSONE CB, GOODWIN PJ. Comorbidities and Their Management: Potential Impact on Breast Cancer Outcomes. Adv Exp Med Biol 2015; 862: 9-21. https://doi.org/10.1007/978-3-319-16366-6_2

[4] SCHÖPF B, WEISSENSTEINER H, SCHÄFER G, FENDT L, GNAIGER E et al. Shift of mitochondrial oxidative phosphorylation is associated with mtDNA mutational load in primary prostate cancer tissue. European Urology Supplements 2018; 17: e85. https://doi.org/10.1016/S15699056(18)30911-4

[5] KOPPENOL WH, BOUNDS PL, DANG CV. Otto Warburg's contributions to current concepts of cancer metabolism. Nat Rev Cancer 2011; 11: 325-337. https://doi.org/10.1038/ $\operatorname{nrc} 3038$

[6] BRAND KA, HERMFISSE U. Aerobic glycolysis by proliferating cells: a protective strategy against reactive oxygen species. FASEB J 1997; 11: 388-395. https://doi.org/10.1096/ fasebj.11.5.9141507

[7] DEBLOIS G, ST-PIERRE J, GIGUERE V. The PGC-1/ERR signaling axis in cancer. Oncogene 2013; 32: 3483-3490. https://doi.org/10.1038/onc.2012.529

[8] YANG H, YANG R, LIU H, REN Z, KONG F et al. Synergism between PGC-1 $\alpha$ and estrogen in the survival of endometrial cancer cells via the mitochondrial pathway. Onco Targets Ther 2016; 9: 3963-3973. https://doi.org/10.2147/ OTT.S103482

[9] YANG H, YANG R, LIU H, REN Z, WANG C et al. Knockdown of peroxisome proliferator-activated receptor gamma coactivator-1 alpha increased apoptosis of human endometrial cancer HEC-1A cells. Onco Targets Ther 2016; 9: 53295338. https://doi.org/10.2147/OTT.S102816

[10] LEBLEU VS, O'CONNELL JT, GONZALEZ HERRERA KN, WIKMAN H, PANTEL K et al. PGC-1 $\alpha$ mediates mitochondrial biogenesis and oxidative phosphorylation in cancer cells to promote metastasis. Nat Cell Biol 2014; 16: 992-1003, 1-15. https://doi.org/10.1038/ncb3039

[11] YUN SH, HAN SH, PARK JI. Peroxisome proliferatoractivated receptor $\gamma$ and PGC-1 $\alpha$ in cancer: dual actions as tumor promoter and suppressor. PPAR Res 2018; 2018: 6727421. https://doi.org/10.1155/2018/6727421

[12] TORRANO V, VALCARCEL-JIMENEZ L, CORTAZAR AR, LIU X, UROSEVIC J et al. The metabolic co-regulator PGC1 $\alpha$ suppresses prostate cancer metastasis. Nat Cell Biol 2016; 18: 645-656. https://doi.org/10.1038/ncb3357

[13] KAMINSKIL, TORRINO S, DUFIES M, DJABARIZ, HAIDER R et al. PGC-1 $\alpha$ inhibits polyamine synthesis to suppress prostate cancer aggressiveness. Cancer Res 2019; 79: 32683280. https://doi.org/10.1158/0008-5472.CAN-18-2043
[14] JEONG SM, HAIGIS MC. Sirtuins in Cancer: a Balancing Act between Genome Stability and Metabolism. Mol Cells 2015; 38: 750-758. https://doi.org/10.14348/molcells.2015.0167

[15] MARTINEZ-PASTOR B, MOSTOSLAVSKY R. Sirtuins, metabolism, and cancer. Front Pharmacol 2012; 3: 22. https://doi.org/10.3389/fphar.2012.00022

[16] TORRENS-MAS M, OLIVER J, ROCA P, SASTRE-SERRA J. SIRT3: Oncogene and Tumor Suppressor in Cancer. Cancers (Basel) 2017; 9: 90. https://doi.org/10.3390/cancers 9070090

[17] LI H, FENG Z, WU W, LI J, ZHANG J et al. SIRT3 regulates cell proliferation and apoptosis related to energy metabolism in non-small cell lung cancer cells through deacetylation of NMNAT2. Int J Oncol 2013; 43: 1420-1430. https://doi. org/10.3892/ijo.2013.2103

[18] CUI Y, QIN L, WU J, QU X, HOU C et al. SIRT3 Enhances Glycolysis and Proliferation in SIRT3-Expressing Gastric Cancer Cells. PLoS One 2015; 10: e0129834. https://doi. org/10.1371/journal.pone.0129834

[19] RODGERS JT, LERIN C, HAAS W, GYGI SP, SPIEGELMAN BM et al. Nutrient control of glucose homeostasis through a complex of PGC-1alpha and SIRT1. Nature 2005; 434: 113-118. https://doi.org/10.1038/nature03354

[20] SHI T, WANG F, STIEREN E, TONG Q. SIRT3, a mitochondrial sirtuin deacetylase, regulates mitochondrial function and thermogenesis in brown adipocytes. J Biol Chem 2005; 280: 13560-13567. https://doi.org/10.1074/jbc.M414670200

[21] WU Y, LU H, YANG H, LI C, SANG Q et al. Zinc stimulates glucose consumption by modulating the insulin signaling pathway in L6 myotubes: essential roles of Akt-GLUT4, GSK3 $\beta$ and mTOR-S6K1. J Nutr Biochem 2016; 34: 126135. https://doi.org/10.1016/j.jnutbio.2016.05.008

[22] QIUYU Q, XING M, YANLI Z. Targeted delivery of doxorubicin to mitochondria using mesoporous silica nanoparticle nanocarriers. Nanoscale 2015; 7: 16677-16686. https://doi. org/10.1039/c5nr05139h

[23] CHEN W, ZHENG R, ZENG H, ZHANG S, HE J. Annual report on status of cancer in China, 2011. Chin J Cancer Res 2015; 27: 2-12. https://doi.org/10.3978/j.issn.10009604.2015.01.06

[24] WARBURG O. On the origin of cancer cells. Science 1956; 123: 309-314. https://doi.org/10.1126/science.123.3191.309

[25] WALLACE DC. Mitochondria and cancer: Warburg addressed. Cold Spring Harb Symp Quant Biol 2005; 70: 363374. https://doi.org/10.1101/sqb.2005.70.035

[26] PUIGSERVER P, WU Z, PARK CW, GRAVES R, WRIGHT $\mathrm{M}$ et al. A cold-inducible coactivator of nuclear receptors linked to adaptive thermogenesis. Cell 1998; 92: 829-839. https://doi.org/10.1016/s0092-8674(00)81410-5

[27] DE MOURA MB, DOS SANTOS LS, VAN HOUTEN B. Mitochondrial dysfunction in neurodegenerative diseases and cancer. Environ Mol Mutagen 2010; 51: 391-405. https:// doi.org/10.1002/em.20575

[28] SHIOTA M, YOKOMIZO A, TADA Y, INOKUCHI J, TATSUGAMI K et al. Peroxisome proliferator-activated receptor gamma coactivator-1alpha interacts with the androgen receptor (AR) and promotes prostate cancer cell growth by activating the AR. Mol Endocrinol 2010; 24: 114-127. https://doi.org/10.1210/me.2009-0302 
[29] LEBLEU VS, O'CONNELL JT, GONZALEZ HERRERA KN, WIKMAN H, PANTEL K et al. PGC-1alpha mediates mitochondrial biogenesis and oxidative phosphorylation in cancer cells to promote metastasis. Nat Cell Biol 2014; 16 : 992-1003, 1-15. https://doi.org/10.1038/ncb3039

[30] LIN J, HANDSCHIN C, SPIEGELMAN BM. Metabolic control through the PGC-1 family of transcription coactivators. Cell Metab 2005; 1: 361-370. https://doi.org/10.1016/j. cmet.2005.05.004

[31] WATKINS G, DOUGLAS-JONES A, MANSEL RE, JIANG WG. The localisation and reduction of nuclear staining of PPARgamma and PGC-1 in human breast cancer. Oncol Rep 2004; 12: 483-488

[32] JIANG WG, DOUGLAS-JONES A, MANSEL RE. Expression of peroxisome-proliferator activated receptor-gamma (PPARgamma) and the PPARgamma co-activator, PGC-1, in human breast cancer correlates with clinical outcomes. Int J Cancer 2003; 106: 752-757. https://doi.org/10.1002/ ijc. 11302

[33] AUDET-WALSH É, PAPADOPOLI D, GRAVEL SP, YEE T, BRIDON G et al. The PGC-1a/ERRa Axis Represses One-Carbon Metabolism and Promotes Sensitivity to Antifolate Therapy in Breast Cancer. Cell Rep 2016; 14: 920-931. https://doi.org/10.1016/j.celrep.2015.12.086

[34] LAGORY EL, WU C, TANIGUCHI CM, DING CKC, CHI JT et al. Suppression of PGC-1 $\alpha$ is critical for reprogramming oxidative metabolism in renal cell carcinoma. Cell Rep 2015; 12: 116-127. https://doi.org/10.1016/j.celrep.2015.06.006

[35] PAVLOVA NN, THOMPSON CB. The Emerging Hallmarks of Cancer Metabolism. Cell Metab 2016; 23: 27-47. https:// doi.org/10.1016/j.cmet.2015.12.006

[36] QIN Y, CHENG C, LU H, WANG Y. miR-4458 suppresses glycolysis and lactate production by directly targeting hexokinase 2 in colon cancer cells. Biochem Biophys Res Commun 2016; 469: 37-43. https://doi.org/10.1016/j.bbrc.2015.11.066

[37] KANG F, MA W, MA X, SHAO Y, YANG W et al. Propranolol Inhibits Glucose Metabolism and 18F-FDG Uptake of Breast Cancer Through Posttranscriptional Downregulation of Hexokinase-2. J Nucl Med 2014; 55: 439-445. https://doi. org/10.2967/jnumed.113.121327

[38] ISRAELSEN WJ, HEIDEN MGV. Pyruvate kinase: function, regulation and role in cancer. Semin Cell Dev Biol 2015; 43: 43-51. https://doi.org/10.1016/j.semcdb.2015.08.004
[39] YE X, SUN Y, XU Y, CHEN Z, LU S et al. Integrated In Silico-In Vitro Discovery of Lung Cancer-related Tumor Pyruvate Kinase M2 (PKM2) Inhibitors. Med Chem 2016; 12: 613-620. https://doi.org/10.2174/1573406412666160307151 535

[40] SALAMA SA, MOHAMMAD MA, DIAZ-ARRASTIA CR, KAMEL MW, KILIC GS et al. Estradiol-17 $\beta$ upregulates pyruvate kinase M2 expression to coactivate estrogen receptor- $\alpha$ and to integrate metabolic reprogramming with the mitogenic response in endometrial cells. J Clin Endocrinol Metab 2014; 99: 3790-3799. https://doi.org/10.1210/ jc.2013-2639

[41] ALHAZZAZI TY, KAMARAJAN P, VERDIN E, KAPILA YL. SIRT3 and cancer: Tumor promoter or suppressor? Biochim Biophys Acta 2011; 1816: 80-88. https://doi. org/10.1016/j.bbcan.2011.04.004

[42] ASHRAF N, ZINO S, MACINTYRE A, KINGSMORE D, PAYNE AP et al. Altered sirtuin expression is associated with node-positive breast cancer. Br J Cancer 2006; 95: 10561061. https://doi.org/10.1038/sj.bjc.6603384

[43] ALHAZZAZI TY, KAMARAJAN P, JOO N, HUANG JY, VERDIN E et al. Sirtuin-3 (SIRT3), a novel potential therapeutic target for oral cancer. Cancer 2011; 117: 1670-1678. https://doi.org/10.1002/cncr.25676

[44] CHEN Y, FU LL, WEN X, WANG XY, LIU J et al. Sirtuin-3 (SIRT3), a therapeutic target with oncogenic and tumor-suppressive function in cancer. Cell Death Dis 2014; 5: e1047. https://doi.org/10.1038/cddis.2014.14

[45] LIU SG, WANG YM, ZHANG YJ, HE XJ, MA T et al. ZL006 protects spinal cord neurons against ischemia-induced oxidative stress through AMPK-PGC-1 $\alpha$-Sirt3 pathway. Neurochem Int 2017; 108: 230-237. https://doi.org/10.1016/j. neuint.2017.04.005

[46] XIAOYAN Y, LI Z, XIUYING Y, HUANG H, HUANG Z et al. Salvianolic acid A protects the peripheral nerve function in diabetic rats through regulation of the AMPK-PGC1aSirt3 axis. Molecules 2012; 17: 11216-11228. https://doi. org/10.3390/molecules170911216

[47] KONG X, WANG R, XUE Y, LIU X, ZHANG H et al. Sirtuin 3, a New Target of PGC-1a, Plays an Important Role in the Suppression of ROS and Mitochondrial Biogenesis. PLoS One 2010; 5: e11707. https://doi.org/10.1371/journal. pone.0011707 


\section{PGC-1 $\alpha$ activates SIRT3 to modulate cell proliferation and glycolytic metabolism in breast cancer}

Yan ZU ${ }^{1, *}$, Xiao-Fei CHEN ${ }^{2}$, Qiang LI ${ }^{1}$, Shu-Ting ZHANG ${ }^{1}$, Li-Na SI ${ }^{1}$

Supplementary Information

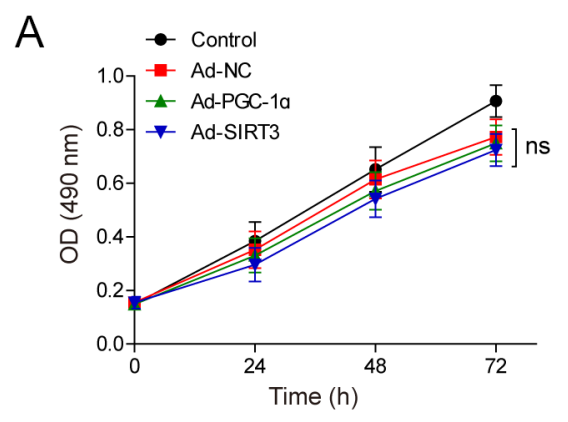

C

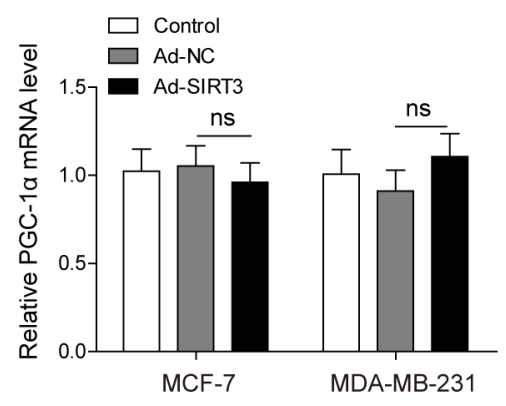

B

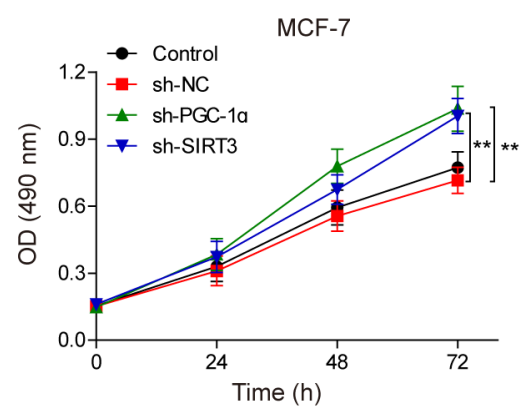

MCF-7

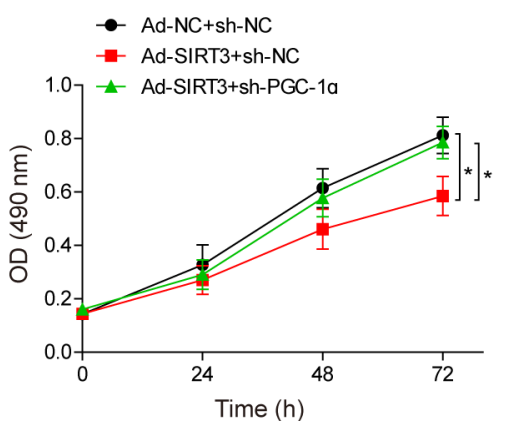

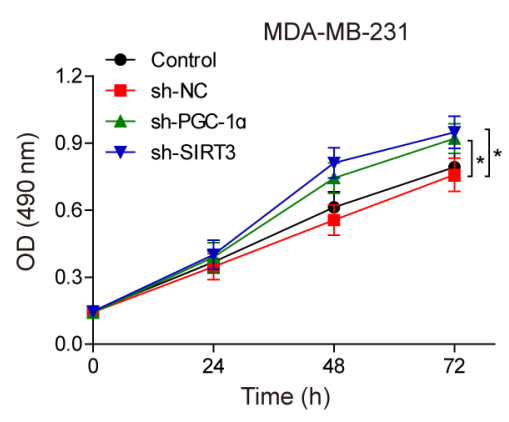

MDA-MB-231

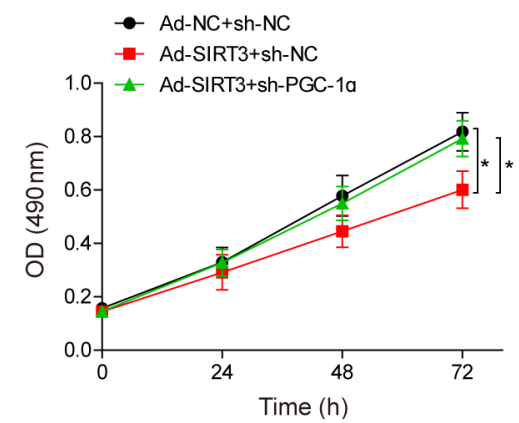

Supplementary Figure S1. A) CCK-8 assay was performed to assess the cell viability of PGC-1 $\alpha$ or SIRT3 overexpression on the normal cell line MCF10A cells. B) The effects of PGC-1 $\alpha$ or SIRT3 knockdown on cell viability were examined using the CCK-8 assay. C) Cell viability was determined by the CCK-8 assay. D) qRT-PCR assay was subjected to evaluate the expression of PGC-1a within SIRT3 overexpression. Data are presented as mean \pm SD. Statistical significance compared with control, ${ }^{*} \mathrm{p}<0.05,{ }^{* *} \mathrm{p}<0.01$. 